\title{
Intratumoral tertiary lymphoid organ is a favourable prognosticator in patients with pancreatic cancer
}

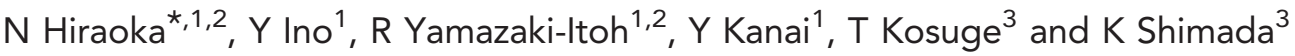 \\ ${ }^{1}$ Division of Molecular Pathology, National Cancer Center Research Institute, Tokyo 104-0045, Japan; ${ }^{2}$ Division of Pathology and \\ Clinical Laboratories, National Cancer Center Hospital, 5-1-1 Tsukiji, Chuo-ku, Tokyo 104-0045, Japan and ${ }^{3}$ Hepato-Biliary and \\ Pancreatic Surgery Division, National Cancer Center Hospital, Tokyo 104-0045, Japan
}

Background: Host immunity has critical roles in tumour surveillance. Tertiary lymphoid organs (TLOs) are induced in various inflamed tissues. The aim of this study was to investigate the clinicopathological and pathobiological characteristics of tumour microenvironment in pancreatic ductal carcinoma (PDC) with TLOs.

Methods: We examined 534 PDCs to investigate the clinicopathological impact of TLOs and their association with tumourinfiltrating immune cells, the cytokine milieu, and tissue characteristics.

Results: There were two different localisations of PDC-associated TLOs, intratumoral and peritumoral. A better outcome was observed in patients with intratumoral TLOs, and this was independent of other survival factors. The PDC tissues with intratumoral TLOs showed significantly higher infiltration of $T$ and B cells and lower infiltration of immunosuppressive cells, as well as significantly higher expression of Th1- and Th17-related genes. Tertiary lymphoid organs developed with an association with arterioles, venules, and nerves. These structures were reduced in an association with cancer invasion in PDC tissues, except for those with intratumoral TLOs. The PDC tissues with intratumoral TLOs had capillaries consisting of mature endothelial cells covered by pericytes.

Conclusions: Our results suggest that the presence of intratumoral TLOs represents a microenvironment that has an active immune reaction, and shows a relatively intact vascular network retained.

Pancreatic cancer (pancreatic ductal carcinoma (PDC)) is the fourth and fifth leading cause of cancer-related death in the United States and Japan, respectively (Center for Cancer Control and Information Services and National Cancer Center J, 2009; Siegel et al, 2011). As PDC shows aggressive growth and early metastatic dissemination, the overall 5-year survival rate for patients with pancreatic cancer is $3-5 \%$, and that of patients treated by curative resection is $15-25 \%$ (Hruban et al, 2010; Siegel et al, 2011). It is necessary to understand the pathobiological characteristics of the PDC tissue more profoundly. In addition, the development of biomarkers to assist the selection of patient subsets is useful for studies aimed at reducing the mortality of PDC patients, especially in phase clinical studies evaluating various therapeutic approaches.

The host immune system is one of the leading players in the tumour microenvironment (Hanahan and Weinberg, 2011), and has a critical role in tumour surveillance. The presence of high numbers of tumour-infiltrating lymphocytes has been found to be a major predictor of favourable clinical outcome in several solid cancers, such as colorectal, lung, and ovarian cancers (Fridman et al, 2012). In pancreatic cancers, it has been shown that several subsets of tumour-infiltrating lymphocytes, neutrophils, and macrophages are independent predictors of a favourable or poor outcome (Fukunaga et al, 2004; Hiraoka et al, 2006; Ino et al, 
2013). Accumulated evidence has indicated that even the same types or subsets of tumour-infiltrating immune cells sometimes have different and opposite effects on patient outcome (Fridman et al, 2012; Remark et al, 2013). These outcomes are probably due to differences in the characteristics of the tissues from which cancers develop, the type of cancer itself, and interaction of the two. Therefore, it is important to understand the tumour microenvironments in different types of cancer.

Tertiary lymphoid organs (TLOs) or structures (alternatively, ectopic lymphoid structures) can develop in various kinds of inflamed and non-lymphoid tissues, including chronic infections, autoimmune diseases, chronic allograft rejection, and several solid cancers (Carragher et al, 2008; Hayasaka et al, 2010; van de Pavert and Mebius, 2010). Tertiary lymphoid organs are thought to have roles in the immune system similar to secondary lymphoid organs (SLOs). Tertiary lymphoid organs are organised lymphoid structures similar to SLOs such as lymph nodes and Peyer's patches, characterised by B-cell follicles, T-cell zones, and specialised vessels known as high endothelial venules (HEVs), although TLOs are not encapsulated and supplied by afferent lymphatics. Large numbers of lymphocytes accumulate in TLOs by homing through the HEVs from the blood by a multistep mechanism that involves L-selectin-, chemokine-, and integrinmediated lymphocyte endothelial cell interaction (von Andrian and Mempel, 2003; Miyasaka and Tanaka, 2004; Girard et al, 2012). High endothelial venules specifically express L-selectin ligands, peripheral node addressin (PNAd), that are sulphated sialyl Lewis X (Hiraoka et al, 1999; Yeh et al, 2001) and chemokines CCL19, CCL21, and CXCL13. CCL19 and CCL21 are necessary for the recruitment of T cells and dendritic cells, and CXCL13 functions in the recruitment of B cells. These chemokines are also involved in lymphoid neogenesis (Carragher et al, 2008; Hayasaka et al, 2010; van de Pavert and Mebius, 2010).

Tertiary lymphoid organs are generally induced under local activation of immune responses, and their presence is reportedly correlated with a better prognosis in breast, lung, and colorectal cancers (Dieu-Nosjean et al, 2008; Coppola et al, 2011; Martinet et al, 2011). However, it is still unclear where TLOs develop in cancer tissues, whether TLOs are associated with any tissue structures including vascular system, and whether TLOs are affected by cancer invasion. It has been shown that functional vascular system is important for antitumour immune response in mouse model, although few studies showing it in human cancer have been reported (Hamzah et al, 2008; Carmeliet and Jain, 2011).

In the present study, we investigated the clinicopathological characteristics of TLOs in PDC and their association with tumourinfiltrating immune cells, the tumour cytokine milieu, and tumour tissue characteristics. We found that there are two different localisation for TLOs associated with PDC - intratumoral and peritumoral - and that the presence of intratumoral TLOs is significantly correlated with an active immune reaction and a favourable patient outcome. We also found that TLOs exist in close association with arterioles, venules, and peripheral nerve fibres that were not invaded by cancer cells and remain in the cancer tissues. It is suggested that lower cancer invasiveness to retain relatively intact vascular network and a host immune reaction are involved in the induction of intratumoral TLOs in PDC tissue.

\section{MATERIALS AND METHODS}

Patients and samples. This study was approved by the National Cancer Center Institutional Review Board. Clinical and pathological data were obtained through a detailed retrospective review of the medical records of all 308 consecutive patients with PDC who had undergone initial surgical resection between 1990 and 2004 at the National Cancer Center Hospital, Japan. None of the patients had received any therapy before surgery. All of the patients included in this study underwent macroscopic curative resection. All of the cases were conventional ductal carcinomas; adenocarcinomas originating in intraductal papillary mucinous neoplasms or mucinous cystic neoplasms were excluded, as were secondary

Table 1. Correlation of intratumoral TLO with clinicopathological characteristics

\section{Intratumoral TLOs}

\begin{tabular}{|l|c|r|r|l|}
\hline Characteristics & No. of patients & Absence & Presence & $P$-value \\
\hline Age (years) & 110 & 92 & 18 & \\
\hline$<60$ & 198 & 167 & 31 & 0.872 \\
$\geq 60$ & 186 & 153 & 33 & \\
\hline Sex & 122 & 106 & 16 & 0.340 \\
\hline Male
\end{tabular}

Size $(\mathrm{mm})$

\begin{tabular}{|l|r|r|r|r|}
\hline$<30$ & 75 & 61 & 14 & \\
$\geq 30$ & 233 & 198 & 35 & 0.470 \\
\hline
\end{tabular}

\section{Tumour margin status}

\begin{tabular}{|l|r|r|r|l|}
\hline Negative & 214 & 183 & 31 & \\
Positive & 94 & 76 & 18 & 0.313 \\
\hline
\end{tabular}

\begin{tabular}{|l|r|r|r|l|}
\hline \multicolumn{5}{|l|}{ Pathologic tumour status } \\
\hline T1 & 7 & 4 & 3 & \\
T2 & 2 & 2 & 0 & \\
T3 & 299 & 253 & 46 & \\
T4 & 0 & 0 & 0 & $0.120^{\text {a }}$
\end{tabular}

Pathologic node status

\begin{tabular}{|l|r|r|r|l|}
\hline N0 & 56 & 45 & 11 & \\
N1 & 252 & 214 & 38 & 0.420 \\
\hline
\end{tabular}

Pathologic metastasis status

\begin{tabular}{|l|r|r|r|l|}
\hline M0 & 270 & 224 & 46 & \\
M1 & 38 & 35 & 3 & 0.234 \\
\hline
\end{tabular}

\begin{tabular}{|l|r|r|r|r|}
\hline \multicolumn{5}{|l|}{ Stage } \\
\hline IA & 4 & 2 & 2 & \\
IB & 1 & 1 & 0 & \\
IIA & 51 & 42 & 9 & \\
IIB & 214 & 179 & 35 & \\
III & 0 & 0 & 0 & \multirow{2}{*}{ IV } \\
IV & 38 & 35 & 3 & $0.228^{a}$
\end{tabular}

\begin{tabular}{|l|r|r|r|l|}
\hline \multicolumn{5}{|l|}{ Tumour histological grade } \\
\hline W/D & 88 & 64 & 24 & \\
M/D & 150 & 132 & 18 & \\
P/D & 70 & 63 & 7 & 0.002 $^{\text {a }}$ \\
\hline
\end{tabular}

\begin{tabular}{|c|c|c|c|c|}
\hline \multicolumn{5}{|c|}{ Nerve plexus invasion ${ }^{b}$} \\
\hline Absence & 98 & 81 & 17 & \\
\hline Presence & 210 & 178 & 32 & 0.621 \\
\hline \multicolumn{5}{|c|}{ Lymphatic invasion $^{b}$} \\
\hline 0,1 & 90 & 71 & 19 & \\
\hline 2,3 & 218 & 188 & 30 & 0.124 \\
\hline \multicolumn{5}{|c|}{ Venous invasion $^{\mathrm{b}}$} \\
\hline 0,1 & 116 & 89 & 27 & \\
\hline 2,3 & 192 & 170 & 22 & 0.010 \\
\hline \multicolumn{5}{|c|}{ Intrapancreatic neural invasion ${ }^{b}$} \\
\hline 0,1 & 132 & 110 & 22 & \\
\hline 2,3 & 176 & 149 & 27 & 0.756 \\
\hline
\end{tabular}

Abbreviations: $M / D=$ moderately differentiated tubular adenocarcinoma; $P / D=$ poorly differentiated adenocarcinoma; $W / D=$ well-differentiated tubular adenocarcinoma and papillary carcinoma.

${ }^{\mathrm{a}}$ Comparisons of qualitative variables are performed using the $\chi^{2}$ test, and otherwise the Fisher's exact test.

${ }^{\mathbf{b}}$ Classified according to the classification of pancreatic carcinoma of Japan Pancreas Society. 
tumours and postneoadjuvant cases. Autoimmune pancreatitisassociated cancers were excluded. The clinicopathological characteristics of the patients are summarised in Table 1. Chronic pancreatitis was usually associated with PDC tissue. The median follow-up period after surgery for the patients as a whole and for the living patients were 17.6 (2.6-201) and 65.8 (2.6-201) months, respectively. Every patient was followed up in the outpatient clinic every 1-3 months during the first postoperative year, and every 6-12 months thereafter. Patients underwent physical examination, laboratory tests, chest radiography, abdominal computed tomography, and/or ultrasonography, unless there was a confirmed relapse. The tumour markers carcinoembryonic antigen and carbohydrate antigen 19-9 were also measured until relapse. Recurrence was suspected when a new local or distant metastatic lesion was found on serial images and an increase in tumour marker levels was recognised. When progression of the disease was confirmed by repeated imaging studies, the date of the first suspicious radiologic finding was used as the date of initial disease recurrence. At the census date (September 2011), we checked whether the patients were dead or alive; 46 patients (14.9\%) were alive, 219 (71.1\%) had died of pancreatic cancer, and 43 (14.0\%) had died of other causes. Information on postresection adjuvant therapy was available for 289 patients, of whom 23 received chemotherapy and radiotherapy, 127 chemotherapy only, and two radiotherapy only; 137 patients did not receive any postresection therapy. All M1 (TNM classification) patients had nodal metastasis around the abdominal aorta, without any other form of metastasis.

For the validation study, we used another cohort comprising 226 consecutive patients with PDC who had undergone initial surgical resection between 2005 and 2009 at the National Cancer Center Hospital, Japan. None of the patients had received any therapy before surgery. All of the patients included in this study underwent macroscopic curative resection. All of the cases were conventional ductal carcinomas; intraductal papillary-mucinous
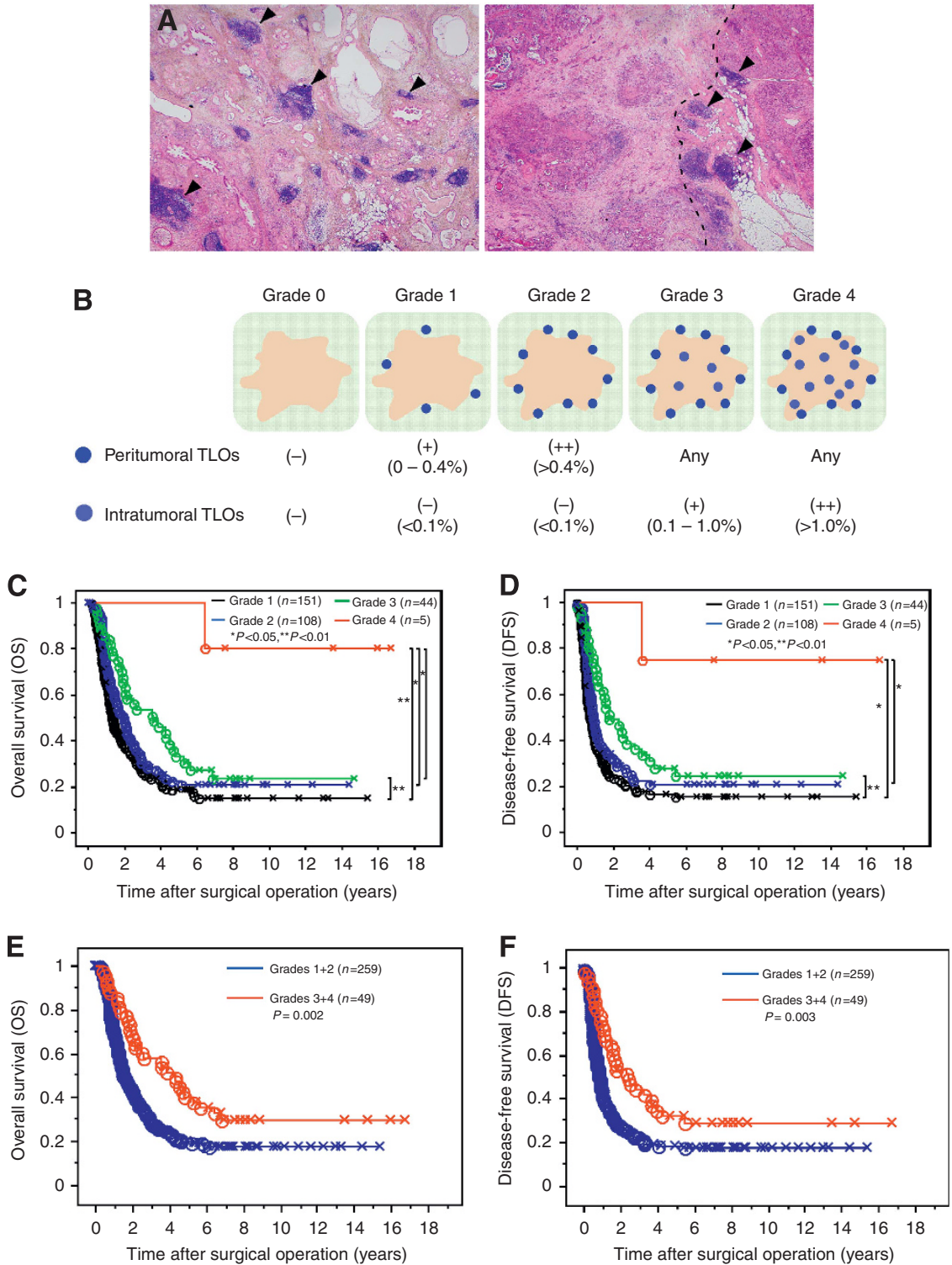

Figure 1. Tertiary lymphoid organs in PDC tissue and their grading. (A) Histology of PDC tissues with TLOs stained with haematoxylin and eosin. There are intratumoral TLOs (arrowheads) in the PDC tissue (left panel) and peritumoral TLOs (arrowheads) surrounding the PDC tissue (right panel) in a low-power view. Dotted line represents the border between PDC and non-cancerous tissue. (B) Tertiary lymphoid organ grading. (C-F) Kaplan-Meier survival curves. Kaplan-Meier survival curves showing comparison of OS among TLO grades (C) and of DFS among TLO grades (D).

Kaplan-Meier survival curves showing comparison of OS between the presence (grades $3+4$ ) and absence (grades $1+2$ ) of intratumoral TLOs in (E) and of DFS between the presence and absence of intratumoral TLOs in (F). The 'circle' and ' $x$ ' represent failure and censoring, respectively. 
neoplasm-related cancers, secondary tumours, and postneoadjuvant cases were excluded. The median follow-up periods after surgery for the patients as a whole and for the living patients were 21.6 (1.9-79.6) and 26.0 (1.9-79.6) months, respectively. At the census date (September 2011), we checked whether all our patients were dead or alive from our medical records and family registers administered by the Japanese government: 90 patients $(39.8 \%)$ were alive, $119(52.7 \%)$ had died of pancreatic cancer, and 17 $(7.5 \%)$ had died of other causes. Information on postresection adjuvant therapy was available for 210 patients, of whom 5 received chemotherapy and radiotherapy, 156 chemotherapy only, and 0 radiotherapy only; 149 patients did not receive any postresection therapy.

Evaluation of TLO and TLO classification. Tertiary lymphoid organs are organised lymphoid structures similar to SLOs such as lymph nodes and Peyer's patches, characterised by B-cell follicles, T-cell zones, and specialised vessels known as HEVs, although TLOs are not encapsulated and supplied by afferent lymphatics (Carragher et al, 2008; Hayasaka et al, 2010; van de Pavert and Mebius, 2010). We defined tumour-associated TLOs as either intratumoral, that is, a TLO located within the PDC tissue, or peritumoral, that is, a TLO located outside the PDC tissue and attached to it. Tumour area was detected by microscopic observation and was marked with ink on tissue slides, and then we evaluated the position of TLOs. If a TLO existed on the line of tumour border, it was counted as a peritumoral TLO.

We classified PDC into five categories (Figure 1B) according to the localisation and frequency of TLO in the maximum cut surface of the tumour tissue: grade 0, no TLO present; grade 1, low frequency of peritumoral TLOs (ratio of the area, tumour: total
TLOs $=100 \%: 0-0.4 \%$ ) and no intratumoral TLO (ratio of the area, tumour: total TLOs $=100 \%:<0.1 \%$ ); grade 2 , high frequency of peritumoral TLOs (ratio of the area, tumour: total TLOs $=100 \%$ : $>0.4 \%$ ) and no intratumoral TLO (ratio of the area, tumour: total TLOs $=100 \%$ : $<0.1 \%$ ); grade 3 , low frequency of intratumoral TLOs (ratio of the area, tumour: total TLOs $=100 \%$ : $0.1-1.0 \%$ ); and grade 4 , high frequency of intratumoral TLOs (ratio of the area, tumour: total TLOs $=100 \%$ : $>1.0 \%)$. No criteria were assigned to peritumoral TLOs in grades 3 and 4 , although all grade 3 or 4 PDCs had a high frequency of peritumoral TLOs. The cutoff value had been set in our preliminary study. The microscopic images were imported as digital photo files using a NanoZoomer Digital Pathology system (Hamamatsu Photonics, Hamamatsu, Japan). We measured the area $\left(\mathrm{mm}^{2}\right)$ of each intratumoral TLO and each peritumoral TLO, and the total area $\left(\mathrm{mm}^{2}\right)$ of the PDC tissue with Image J software (NIH, Bethesda, $\mathrm{MD}$, USA). We then calculated the area ratio of total PDC tissue to the total TLOs. Then, we compared the judgement of TLO grading in 51 consecutive cases of PDC on the basis of optical observation by pathologists to that obtained by calculating the area ratio by computational imaging analysis (Supplementary Figure 1A). The interobserver reproducibility had a $\kappa$-value of 0.73 (substantial agreement) and a weighted $\kappa$-value of 0.94 (almost perfect agreement). Then, we judged the TLO grading of PDC tissues on the basis of optical observation by two pathologists later. These observers were blinded to each other and also not provided with any clinical information on the outcome of the patients. If their judgement of TLO grading to the same case was different, the observers discussed the reasons for the difference and performed regrading or we judged the TLO grading by calculating the area ratio by computational imaging analysis.

Table 2. Univariate and multivariate analyses of prognostic factors associated with (a) overall survival and (b) disease-free survival in patients with ductal carcinoma of the pancreas

\begin{tabular}{|c|c|c|c|c|}
\hline \multirow[b]{2}{*}{ Variables } & \multicolumn{2}{|c|}{ Univariate analysis } & \multicolumn{2}{|c|}{ Multivariate analysis } \\
\hline & $\mathrm{HR}(95 \% \mathrm{Cl})$ & $P$-value & $\mathrm{HR}(95 \% \mathrm{Cl})$ & $P$-value \\
\hline \multicolumn{5}{|l|}{ (a) Overall survival } \\
\hline Age ( $<60$ years $/ \geq 60$ years $)$ & $1.054(0.802-1.386)$ & 0.707 & & \\
\hline Gender (female/male) & $0.936(0.712-1.230)$ & 0.634 & & \\
\hline Tumour size $(<30 \mathrm{~mm} / \geq 30 \mathrm{~mm})$ & $1.884(1.326-2.565)$ & 0.0003 & $1.303(0.918-1.849)$ & 0.138 \\
\hline Pathologic tumour status $(\mathrm{T} 1+\mathrm{T} 2 / \mathrm{T} 3)$ & $6.265(1.555-25.377)$ & 0.010 & & \\
\hline Pathologic node status (NO/N1) & $1.967(1.349-2.868)$ & 0.0004 & $1.506(1.018-2.2277)$ & 0.041 \\
\hline Pathologic metastasis status (M0/M1) & $2.070(1.416-3.025)$ & 0.0002 & $1.745(1.179-2.583)$ & 0.005 \\
\hline Histological grade (W/D/M/D, P/D) ${ }^{a}$ & $1.439(1.075-1.927)$ & 0.015 & $1.379(1.022-1.862)$ & 0.036 \\
\hline Tumour margin status (negative/positive) & $1.414(1.062-1.883)$ & 0.018 & $1.188(0.881-1.601)$ & 0.257 \\
\hline Nerve plexus invasion (absence/presence) & $1.485(1.105-1.995)$ & 0.009 & $1.023(0.737-1.421)$ & 0.891 \\
\hline Lymphatic invasion $(0,1 / 2,3)^{\mathrm{a}}$ & $2.163(1.574-2.970)$ & $<0.0001$ & $1.643(1.177-2.292)$ & 0.004 \\
\hline Venous invasion $(0,1 / 2,3)^{\mathrm{a}}$ & $1.926(1.446-2.565)$ & $<0.0001$ & $1.301(0.955-1.772)$ & 0.095 \\
\hline Intrapancreatic neural invasion $(0,1 / 2,3)^{a}$ & $1.814(1.373-2.397)$ & $<0.0001$ & $1.671(1.256-2.223)$ & 0.0004 \\
\hline Intratumoral TLOs (presence/absence) & $1.800(1.234-2.624)$ & 0.002 & $1.637(1.115-2.403)$ & 0.012 \\
\hline \multicolumn{5}{|l|}{ (b) Disease-free survival } \\
\hline Age $(<60$ years $/ \geq 60$ years $)$ & $1.148(0.868-1.523)$ & 0.339 & & \\
\hline Gender (female/male) & $0.974(0.732-1.297)$ & 0.858 & & \\
\hline Tumour size $(<30 \mathrm{~mm} / \geq 30 \mathrm{~mm})$ & $1.911(1.353-2.699)$ & 0.0002 & $1.340(0.926-1.939)$ & 0.120 \\
\hline Pathologic tumour status $(\mathrm{T} 1+\mathrm{T} 2 / \mathrm{T} 3)$ & $3.881(1.239-12.154)$ & 0.020 & $1.701(0.518-5.584)$ & 0.376 \\
\hline Pathologic node status (NO/N1) & $1.985(1.344-2.932)$ & 0.0006 & $1.465(0.977-2.196)$ & 0.063 \\
\hline Pathologic metastasis status (M0/M1) & $2.473(1.659-3.686)$ & $<0.0001$ & $2.091(1.388-3.152)$ & 0.0004 \\
\hline Histological grade (W/D/M/D, P/D) & $1.435(1.061-1.942)$ & 0.019 & $1.245(0.910-1.703)$ & 0.171 \\
\hline Tumour margin status (negative/positive) & $1.343(0.995-1.811)$ & 0.054 & & \\
\hline Nerve plexus invasion (absence/presence) & $1.367(1.010-1.852)$ & 0.043 & $1.042(0.744-1.459)$ & 0.811 \\
\hline Lymphatic invasion $(0,1 / 2,3)^{a}$ & $2.138(1.533-2.980)$ & $<0.0001$ & $1.591(1.112-2.277)$ & 0.011 \\
\hline Venous invasion $(0,1 / 2,3)^{a}$ & $1.917(1.430-2.571)$ & $<0.0001$ & $1.442(1.057-1.969)$ & 0.021 \\
\hline Intrapancreatic neural invasion $(0,1 / 2,3)^{a}$ & $1.594(1.194-2.128)$ & 0.002 & $1.377(1.024-1.851)$ & 0.034 \\
\hline Intratumoral TLOs (presence/absence) & $1.775(1.209-2.607)$ & 0.003 & $1.611(1.092-2.375)$ & 0.016 \\
\hline
\end{tabular}


Pathological examination, immunohistochemistry, double immunofluorescence, evaluation of cancer invasion to arterioles and venules, extraction of RNA and real-time RT-PCR, and statistical analysis are mentioned in the Supplementary Materials and Methods.

\section{RESULTS}

TLOs in PDC tissue. Tertiary lymphoid organs associated with PDC were found within the tumour tissue (intratumoral TLOs) or located around the tumour tissue or in the peritumoral area (peritumoral TLOs) (Figure 1A). We classified PDC into five categories according to the localisation and frequency of TLOs (see Materials and Methods section and Figure 1B): 49\% (151 of 308) of PDCs were classified as grade 1, 35\% (108 of 308) as grade 2, $14.3 \%$ (44 of 308 ) as grade 3 , and $1.6 \%$ (5 out of 308 ) as grade 4 . No tumours without TLOs (grade 0) were found in our series. Histologically, there were B-cell follicles with germinal centers,
T-cell zones with mature DCs, and HEV, which were arranged in a compartment similar to the organisation of a lymph node, in both intratumoral and peritumoral TLOs. Immunohistochemically (Supplementary Figure 1B), the amounts of $\mathrm{PNAd}^{+} \mathrm{HEV}$ endothelial cells, chemokine CCL $21^{+}$cells, CCL19 ${ }^{+}$cells, and $\mathrm{CXCL} 3^{+}$cells in TLOs did not differ significantly between intratumoral and peritumoral TLOs (data not shown).

Prognostic impact of the presence of intratumoral TLOs. Kaplan-Meier survival analysis showed that the presence of intratumoral TLOs in cancer tissue tended to be associated with longer overall survival (OS) and disease-free survival (DFS) (Figures $1 \mathrm{C}$ and D), especially in cases of grade 4 , which included one case of stage $2 \mathrm{~A}$, three of stage $2 \mathrm{~B}$, and one of stage 4 , and four of these five patients being alive without recurrence. Number of grade 4 cases was not enough for analysing statistically, thus we combined patients and compared two groups, patients having only peritumoral TLOs (grades 1 and 2) and patients having intratumoral TLOs (grades 3 and 4) (Figures $1 \mathrm{E}$ and F). Univariate Cox regression analysis demonstrated a correlation between the
A

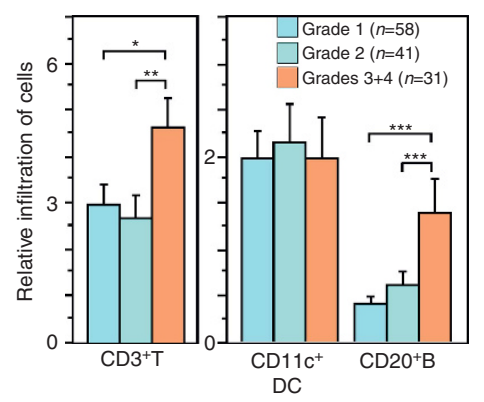

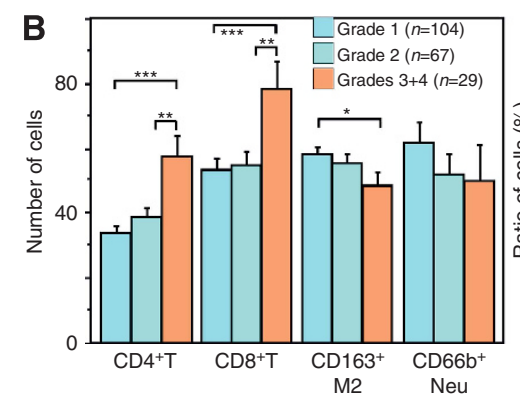

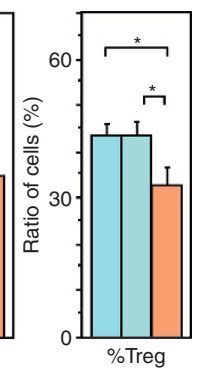

C
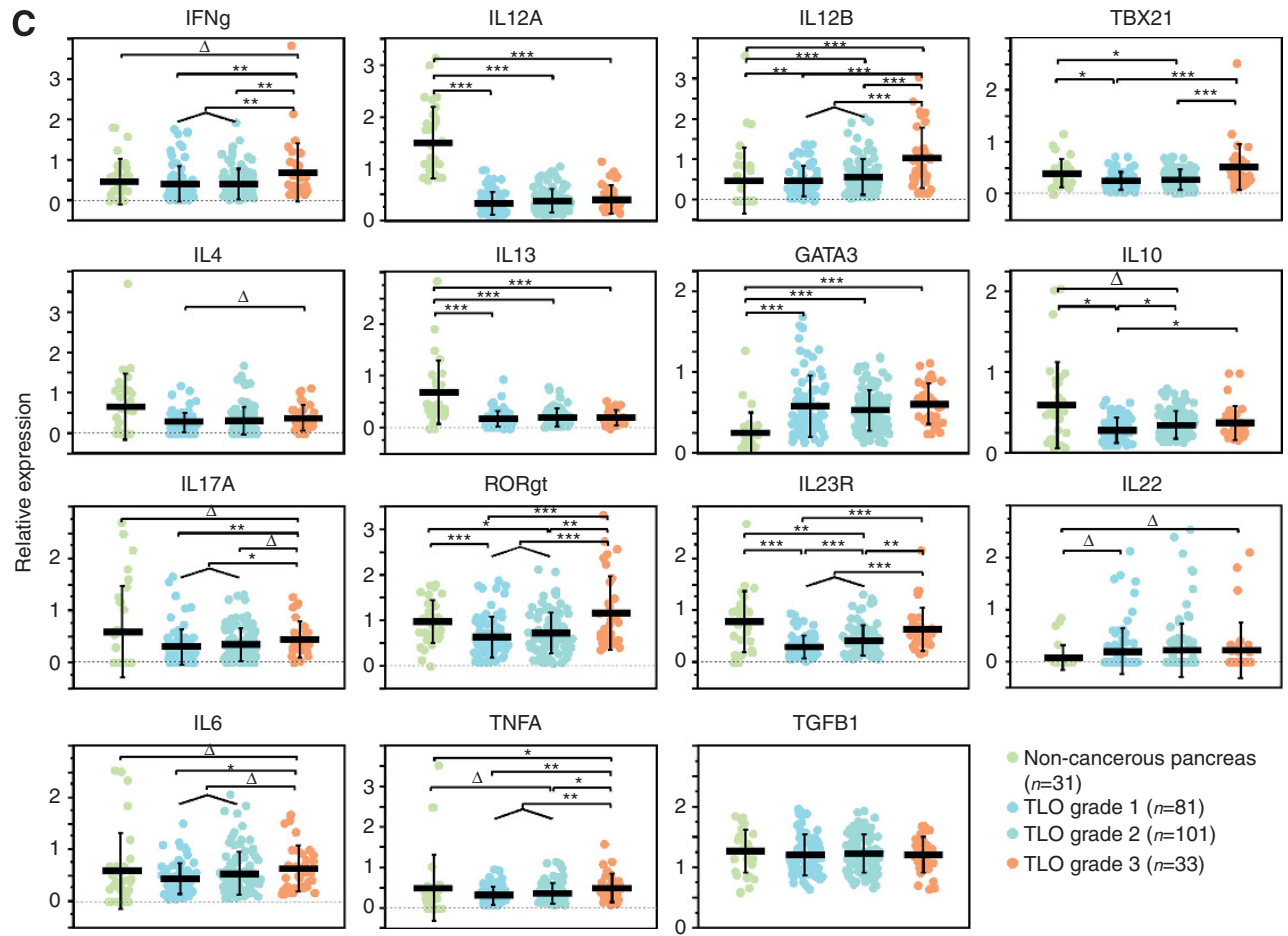

Non-cancerous pancreas

$(n=31)$

TLO grade $1(n=81)$

TLO grade 2 ( $n=101)$

- TLO grade $3(n=33)$

Figure 2. Tumour immune microenvironment of PDC tissues. (A and $\mathbf{B}$ ) Tumour-infiltrating immune cells in PDC tissues of various TLO grades. Ratio (\%) of the total area occupied by the immune-labelled cells relative to the area of the PDC or non-cancerous pancreas tissue in (A). Absolute numbers of tumour-infiltrating immune cells or ratio of tumour-infiltrating immune cells are analysed in PDC tissues in (B). \%Treg represents prevalence of FOXP3 ${ }^{+} \mathrm{CD} 4{ }^{+}$Tregs relative to $\mathrm{CD}^{+}{ }^{+} \mathrm{T}$ cells. Each data column represents the mean value \pm s.e. Significance value (MannWhitney U-test) of $P<0.05\left(^{*}\right), P<0.01\left(^{* *}\right)$, and $P<0.001$ ( $\left.^{* \star *}\right)$. (C) Expression of genes related to the immune microenvironment in PDC tissues of TLO grade $1(n=81)$, grade $2(n=101)$, and grade $3(n=33)$, and in non-cancerous pancreas tissues $(n=31)$, analysed by real-time RT-PCR. Each

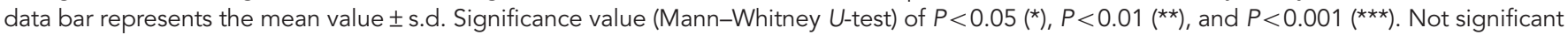
value but having tendency of $P<0.1(\Delta)$. 
presence of intratumoral TLOs and longer OS and DFS (Table 2). The average survival periods for patients having PDC with and without intratumoral TLOs were $48.38 \pm 4.27$ (median 42.67) months and $29.43 \pm 1.62$ (median 15.53) months, respectively. One-year survival rates for patients having PDC with and without intratumoral TLOs were $87.7 \pm 4.7 \%$ and $68.3 \pm 3.0 \%$, respectively; the 2 -year rates were $68.9 \pm 6.8 \%$ and $42.6 \pm 3.2 \%$, and the 5-year rates were $40.4 \pm 7.2 \%$ and $20.2 \pm 2.7 \%$. Multivariate Cox regression analysis showed that the presence of intratumoral TLOs was one of the independent predictors of OS as well as an independent predictor of DFS (Table 2). The prognostic significance of the presence of intratumoral TLOs was confirmed by the validation study using another cohort containing 226 patients with PDC (Supplementary Figures 1C-F and Supplementary Table 1).

When correlations with these clinicopathological features were analysed, the presence of intratumoral TLOs was found to be more likely in cases with more differentiated tumours, and more frequent venous invasion (Table 1).

Relationship between tumour-infiltrating immune cells and TLO grade. Tumour-infiltrating $\mathrm{CD}^{+} \mathrm{T}$ cells and $\mathrm{CD}_{2} 0^{+} \mathrm{B}$ cells were all significantly higher in PDC tissues other than the area of TLOs in cases having intratumoral TLOs (grades 3 and 4) compared with those in cases not having intratumoral TLOs (grades 1 and 2) (Figure 2A). Tumour-infiltrating $\mathrm{CD}^{+}$and $\mathrm{CD}^{+}{ }^{+} \mathrm{T}$ cells in cancer tissues other than the area of TLOs in cases with intratumoral TLOs were also significantly higher compared with those without intratumoral TLOs. Higher tumour-infiltrating $\mathrm{CD}^{+}$and $\mathrm{CD}^{+} \mathrm{T}$ cells and $\mathrm{B}$ cells were shown to provide an antitumour immune microenvironment (Nelson, 2010; Ino et al, 2013). In contrast, the prevalence of tumour-infiltrating $\mathrm{FOXP}^{+}$ regulatory $\mathrm{T}$ cells among $\mathrm{CD} 4{ }^{+} \mathrm{T}$ cells $(\% \mathrm{Treg})$ and $\mathrm{CD} 163^{+} \mathrm{M} 2$ macrophages in cases with intratumoral TLOs was significantly lower compared with those without intratumoral TLOs (Figure 2B). Higher tumour-infiltrating \%Treg, M2 macrophages, or neutrophils were previously shown to be present in an immunetolerant microenvironment (Ino et al, 2013).

Relationship between the cytokine milieu of cancer tissue and TLO grade. We compared gene expression profiles in PDC tissues detected by real-time RT-PCR (Figure 2C). Not all Th1-associated genes, but the IFNg, TBX21, and IL12B genes, showed significantly higher expression in PDC tissues with intratumoral TLOs (grade 3 ) compared with those without intratumoral TLOs (grades 1 and 2). Th17-associated genes (IL17A, RORgt, and IL23R) and inflammation-related genes (IL6 and TNFA) also showed significantly higher expression in PDC tissues with intratumoral TLOs compared with those without intratumoral TLOs. Expression of Th2-associated genes (IL4, IL13, and GATA3) and immune suppression-related genes (IL10 and TGFb1) were comparable between them. These results combined with the results of tumour-infiltrating immune cells suggest that an immune reaction is more active in the tumour microenvironment in PDC tissues with intratumoral TLOs compared with those without intratumoral TLOs. They were also consistent with the microenvironment that upregulation of IL-6 under the presence of TGF- $\beta$ accelerates the differentiation of Th17 and inhibits the induction of Treg. There was little difference in the expression of genes and tumour-infiltrating immune cells between PDC tissues with lower and higher amounts of peritumoral TLOs (grades 1 and 2).

Tissue destruction by cancer cell invasion and its effect on TLOs in PDC tissue. Next, we assessed the relationship between TLOs and tissue structures. Histological observation revealed that TLOs existed in the pancreatic tissue regardless of the localisation, peritumour and intratumour, and were strongly associated with peripheral nerves, arterioles, and venules. Then, we evaluated nerve fibres and vessels in PDC tissues with intratumoral
TLOs compared with tissues of common PDC, most of which are PDCs without intratumoral TLOs (Figures 3 and 4). The density of peripheral nerve fibres was apparently reduced within tissues of common PDC, in comparison with PDC tissues with intratumoral TLOs (Figure 4A, $P<0.0001$ ).

The density of venules or arterioles in PDC tissues tended to reduce when venous or arterial invasion of cancer cells occurred more frequently (Figures $4 \mathrm{~B}$ and $\mathrm{C}$ ). We compared the density of vessels and the frequency of vascular tumour invasion between PDC tissues with intratumoral TLOs and common PDC tissues. The density of paired venules and arterioles within PDC tissues with intratumoral TLOs $\left(0.205 \pm 0.106\right.$ per $\mathrm{mm}^{2}$; median 0.185$)$ was higher compared with that in common PDCs $(0.114 \pm 0.061$ per $\mathrm{mm}^{2}$; median 0.113 ) (Figures $4 \mathrm{~B}$ and $\mathrm{C}, P=0.005$ ). The frequencies of vascular tumour invasion within PDC tissues with intratumoral TLOs (to venules and arterioles, $29.3 \pm 21.0 \%$ (median 24.0) and $1.97 \pm 3.76 \%$ (median 0), respectively) were lower compared with those within common PDCs (to venules and arterioles, $76.0 \pm 25.3 \%$ (median 80 ) and $16.6 \pm 20.1 \%$ (median 8.3), respectively; $P<0.0001$ and $P=0.013$ ) (Figures $4 \mathrm{~B}$ and $\mathrm{C}$ ). These findings were consistent with the fact that intratumoral TLOs were more likely to be present in PDC tissues in cases showing more frequent venous invasion (Table 1).

The PDC tissues with intratumoral TLOs had much more capillaries being retained relatively intact. Next, we investigated

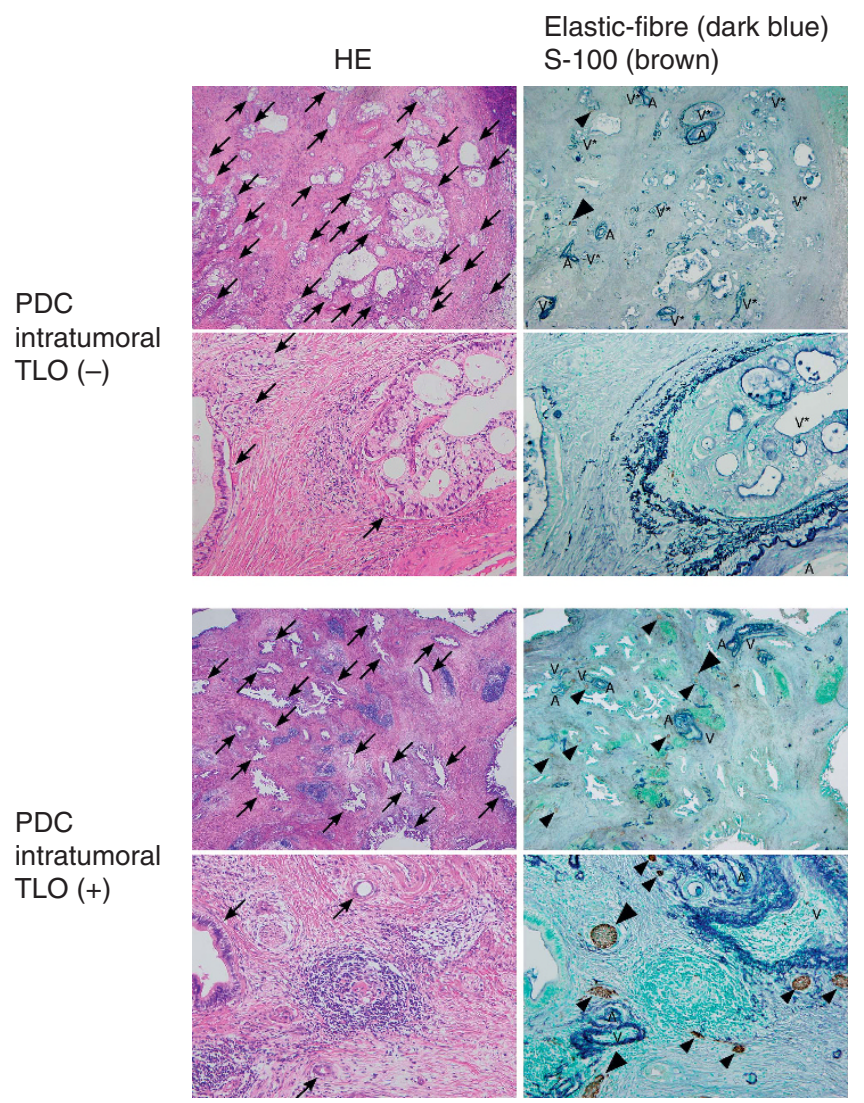

Figure 3. Histology and immunohistochemistry of PDC tissues. Serial sections of tissues affected by the PDC tissue with or without intratumoral TLOs stained with hematoxylin and eosin ( $\mathrm{HE}$, left column), and elastic fibre staining (dark blue) with immunohistochemistry for S-100 (brown) and counterstained with methyl green (right column). Cancer cells (arrows), peripheral nerve fibres (arrowheads), arteriole $\left({ }^{\prime} A\right.$ '), venule (' $V$ '), pancreatic duct (' $D$ '), and a venule infiltrated by cancer cells $\left({ }^{*}{ }^{\star \prime}\right)$ are evident. Upper rows are low-power views and lower rows are medium-power views. 

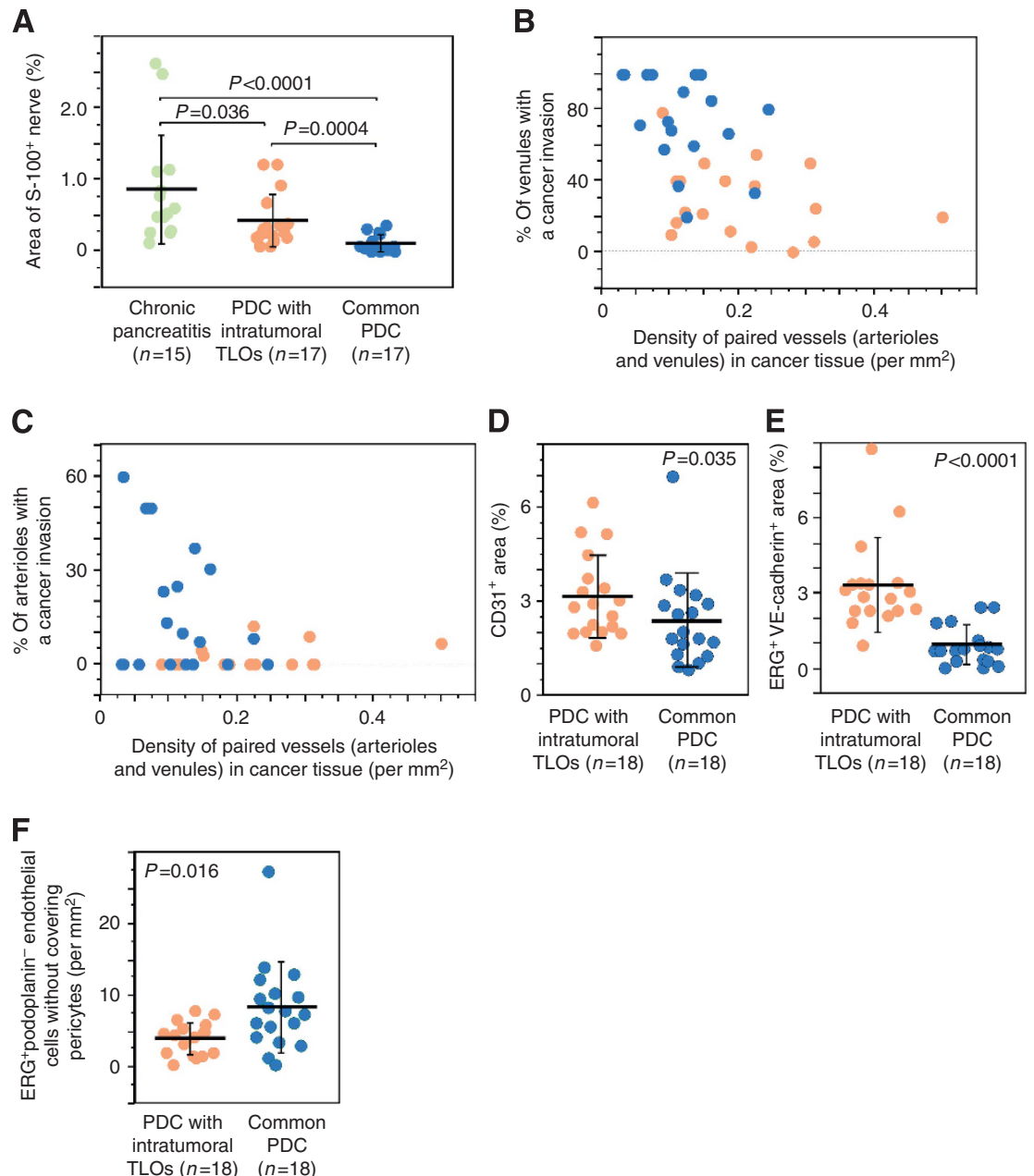

Figure 4. Relationships between TLOs and pancreatic tissue structures. (A) The ratio of the area occupied by S- $100^{+}$nerve fibres relative to the total area of the PDC tissue is compared among tissues affected by chronic pancreatitis, PDC tissues in consecutive cases, and those of cases with intratumoral TLOs. (B) The scatter plot assigning 'the ratio of the venule with venous invasion to the total venules observed with a pair of arterioles' to the horizontal axis and 'the density of venules observed with a pair of arterioles' to the vertical axis is compared between PDC tissues of consecutive cases (blue, $n=18$ ) and those of cases with intratumoral TLOs (orange, $n=18$ ). Significance values are of $P<0.0001$ in the ratio of the venule with venous invasion to the total venules, and of $P=0.005$ in the density of venules. (C) The scatter plot assigning 'the ratio of the arteriole with cancer invasion to the total arterioles observed with a pair of venules' to the horizontal axis and 'the density of arterioles observed with a pair of venules' to the vertical axis is compared between PDC tissues of consecutive cases (blue, $n=18$ ) and those of cases with intratumoral TLOs (orange, $n=18$ ). Significance values are of $P=0.013$ in the ratio of the arteriole with cancer invasion to the total arterioles and of $P=0.005$ in the density of arterioles. (D) The ratio of the area occupied by $\mathrm{CD} 31^{+}$endothelial cells within the total area of the PDC tissue is compared between PDC tissues from consecutive cases and those of cases with intratumoral TLOs. (E) The ratio of the area occupied by ERG ${ }^{+}$VE-cadherin ${ }^{+}$ endothelial cells within the total area of PDC tissue is compared between PDC tissues from consecutive cases and those of cases with intratumoral TLOs. (F) The density of ERG ${ }^{+}$podoplanin ${ }^{-}$endothelial cells composed of abnormal or proliferating blood vessels lacking a covering of $\alpha \mathrm{SMA}^{+}$ pericytes is compared between PDC tissues from consecutive cases $(n=18)$ and those of cases with intratumoral TLOs.

blood vessels that were mainly capillary-level vessels, in addition to arterioles and venules. The number of $\mathrm{CD} 31^{+}$endothelial cells in PDC tissue was higher in cases with intratumoral TLOs compared with that in common PDCs (Figure 4D). The blood vessels were observed abundantly in areas near the TLOs, and such blood vessels showed higher expression of VE-cadherin (Supplementary Figure 2C), which is known to be expressed abundantly in quiescent and mature vessels (Dejana et al, 2009; Sawada et al, 2012). The $\mathrm{ERG}^{+} \mathrm{VE}$-cadherin ${ }^{+}$endothelial cells were significantly richer in the PDC tissue in cases with intratumoral TLOs compared with that in common PDCs (Figure 4E). Conversely, the density of endothelial cells composed of abnormal blood vessels lacking a covering of $\alpha \mathrm{SMA}^{+}$pericytes was higher in common PDCs (Figure 4F and Supplementary Figure 2D). Therefore, although vascular density is usually very low within the PDC tissue (Olive et al, 2009), there were relatively many blood vessels that appeared to be morphologically and immunohistochemically intact around the intratumoral TLOs in the PDC tissue.

\section{DISCUSSION}

We have reported here for the first time that both lower cancer invasiveness, especially to venules, and a host immune reaction are involved in the induction of intratumoral TLOs in cancer tissues. Our investigation, using over 300 cases of PDC, revealed that there were two different localisations of PDC-associated TLOs, intratumoral and peritumoral, the former being relatively rare $(15.9 \%)$ but the latter almost ubiquitous. Univariate and multivariate survival analyses revealed that the presence of intratumoral TLOs in PDC tissue was independently prognostic of OS and DFS. These findings were confirmed by our validation study where over 200 cases of 
PDC were enrolled. Surprisingly, we found a small population (1.5\% (8 of 534)) comprising two patients at stage $2 \mathrm{~A}$, four at stage $2 \mathrm{~B}$ and two at stage 4 who showed a very good outcome among PDC cases with abundant intratumoral TLOs (grade 4), and all but one of these patients were alive without recurrence. The antitumour microenvironment present in PDC tissues with intratumoral TLOs was suggested to be in an active state of cellular immune reaction and B-cell reaction, which was determined by tumour-infiltrating immune cells and the tumour cytokine milieu (Figure 2). Tumour-associated lymphoid neogenesis in the PDC tissue was correlated with the antitumour microenvironment, similar to previous observations of breast cancer (Martinet et al, 2011), lung cancer (Dieu-Nosjean et al, 2008), and malignant melanoma (Cipponi et al, 2012), where the presence of TLOs in cancer tissues was correlated with an active cellular immune reaction and B-cell reaction. These previous studies also showed that an abundance of tumour-associated TLOs including intratumoral and peritumoral TLOs was significantly correlated with longer patient survival. A similar relationship was found in our series. Pancreatic ductal carcinoma cases showing less abundant TLOs showed a significantly shorter OS $(P=0.004)$ and DFS $(P=0.005)$ compared with those with more abundant TLOs, when we performed survival analysis to compare PDC cases with grade 1 TLOs and those with grades 2-4 TLOs, as most of the cases with intratumoral TLOs (grades 3 and 4) had abundant peritumoral TLOs. However, no significant differences were obtained between grade 1 and grade 2 PDC cases in terms of patient outcome, tumour-infiltrating immune cells, and the tumour cytokine milieu. Therefore, the presence of intratumoral TLOs is a more useful hallmark representing an active immune response in the tumour microenvironment, and is an independent prognosticator.

Here, we found that TLOs in the pancreatic tissue associated strongly with arterioles, venules, and peripheral nerve fibres. Tissue destruction and remodelling due to chronic inflammation often obstructed the venules, although the elastic fibres of the venules were retained, and the TLOs had developed in association with them (data not shown). In contrast, no TLOs were found in most of PDC tissues in which these structures had been depleted or reduced, probably owing to cancer invasion followed by tissue remodelling (Figures $4 \mathrm{~B}$ and $\mathrm{C}$ ), suggesting that such cancer invasion destroys some components that is necessary for the development of TLOs in the PDC tissue. The desmoplastic stromal response is capable of affecting the vascularity and vessel function in PDC tissue, although only the desmoplastic response that was also found in chronic pancreatitis did not usually deplete the structures necessary for TLO formation. The mechanism responsible for the development and maintenance of TLOs remains poorly characterised, except with regard to the development of SLOs at the embryonic stage. Although the venules are suggested to be necessary for TLOs, we still lack evidence for any role of arterioles and/or peripheral nerves in this context.

In this study, we noticed that PDC tissues with intratumoral TLOs had relatively large amounts of capillary vessels consisting of $\mathrm{ERG}^{+} \mathrm{VE}^{-}$-cadherin ${ }^{+}$endothelial cells covered by pericytes that seemed to be morphologically and immunohistochemically intact (Figures 4D-F), although the vascular density was exceptionally low within the PDC tissue in general (Olive et al, 2009), suggesting that at least partly functional vascular networks are retained, transporting immune cells or other molecules into the cancer tissues, thereby rendering the antitumour immune reaction more effective. Murine vascular studies have shown that vascular normalisation in tumours enhances the influx of immune effector cells into the tumour parenchyma and markedly prolongs the survival of tumour-bearing mice (Hamzah et al, 2008; Carmeliet and Jain, 2011). This suggests that PDC cases with intratumoral TLOs might offer a higher chance of effector immune cells, drugs, or effector molecules coming into contact with cancer cells subjected to immunotherapy, chemotherapy, or molecular targeting therapy.

Although the clinical significance of tumour-infiltrating Th17 in various types of cancer is controversial (Fridman et al, 2012), more marked infiltration of Th17 into PDC tissues is reportedly associated with a better prognosis in murine tumour models (Gnerlich et al, 2010). Although recently it has been reported that Th17 cells and Th17 cytokines also contribute to the development of TLOs in murine models of chronic inflammation (Peters et al, 2011; Rangel-Moreno et al, 2011), indeed Th17 cells share many developmental and effector markers with lymphoid-tissue inducer (LTi) cells and their related innate lymphoid cells (Spits et al, 2013); LTi cells are critical to the development of SLOs. It is possible that Th17 or LTi-like innate immune cells expressing Th17-related genes contribute to the antitumour immune response directly or through the induction of TLOs. The next focus of interest is how Th17 cytokines act in PDC tissues.

In conclusion, the presence of intratumoral TLOs in PDC tissues appears to be an independent prognosticator and it is suggested that it also represents a microenvironment that is less vulnerable to cancer invasiveness, being associated with an active immune reaction, and relatively intact arterioles, venules, and nerves with vascular networks. It is also suggested that the presence of intratumoral TLOs is a useful hallmark to stratify PDCs by specific tumour microenvironment and to assist the selection of patient subsets for clinical studies evaluating various therapeutic approaches.

\section{ACKNOWLEDGEMENTS}

This work was supported by a Grant-in-Aid for Scientific Research from the Ministry of Education, Culture, Sports, Science, and Technology of Japan (NH), Princess Takamatsu Foundation (1024216) (NH), Takeda Science Foundation (NH), and Cancer Research and Development Fund (NH). The authors have no conflicting financial, professional, or personal interests. We thank Ms Keiko Gomisawa for excellent technical helps and Drs Hidenori Ojima, Minoru Esaki, Satoshi Nara, and Yoji Kishi for useful discussion.

\section{REFERENCES}

Carmeliet P, Jain RK (2011) Principles and mechanisms of vessel normalization for cancer and other angiogenic diseases. Nat Rev Drug Discov 10: 417-427.

Carragher DM, Rangel-Moreno J, Randall TD (2008) Ectopic lymphoid tissues and local immunity. Semin Immunol 20: 26-42.

Center for Cancer Control and Information Services and National Cancer Center J (2009) Cancer Statistics in Japan. Foundation for Promotion of Cancer Research: Tokyo, Japan.

Cipponi A, Mercier M, Seremet T, Baurain JF, Theate I, van den Oord J, Stas M, Boon T, Coulie PG, van Baren N (2012) Neogenesis of lymphoid structures and antibody responses occur in human melanoma metastases. Cancer Res 72: 3997-4007.

Coppola D, Nebozhyn M, Khalil F, Dai H, Yeatman T, Loboda A, Mule JJ (2011) Unique ectopic lymph node-like structures present in human primary colorectal carcinoma are identified by immune gene array profiling. Am J Pathol 179: 37-45.

Dejana E, Tournier-Lasserve E, Weinstein BM (2009) The control of vascular integrity by endothelial cell junctions: molecular basis and pathological implications. Dev Cell 16: 209-221.

Dieu-Nosjean MC, Antoine M, Danel C, Heudes D, Wislez M, Poulot V, Rabbe N, Laurans L, Tartour E, de Chaisemartin L, Lebecque S, Fridman WH, Cadranel J (2008) Long-term survival for patients with non-small-cell lung cancer with intratumoral lymphoid structures. J Clin Oncol 26: 4410-4417. 
Fridman WH, Pages F, Sautes-Fridman C, Galon J (2012) The immune contexture in human tumours: impact on clinical outcome. Nat Rev Cancer 12: 298-306.

Fukunaga A, Miyamoto M, Cho Y, Murakami S, Kawarada Y, Oshikiri T, Kato K, Kurokawa T, Suzuoki M, Nakakubo Y, Hiraoka K, Itoh T, Morikawa T, Okushiba S, Kondo S, Katoh H (2004) CD8 + tumorinfiltrating lymphocytes together with $\mathrm{CD} 4+$ tumor-infiltrating lymphocytes and dendritic cells improve the prognosis of patients with pancreatic adenocarcinoma. Pancreas 28: e26-e31.

Girard JP, Moussion C, Forster R (2012) HEVs, lymphatics and homeostatic immune cell trafficking in lymph nodes. Nat Rev Immunol 12: 762-773.

Gnerlich JL, Mitchem JB, Weir JS, Sankpal NV, Kashiwagi H, Belt BA, Porembka MR, Herndon JM, Eberlein TJ, Goedegebuure P, Linehan DC (2010) Induction of Th17 cells in the tumor microenvironment improves survival in a murine model of pancreatic cancer. J Immunol 185: 4063-4071.

Hamzah J, Jugold M, Kiessling F, Rigby P, Manzur M, Marti HH, Rabie T, Kaden S, Grone HJ, Hammerling GJ, Arnold B, Ganss R (2008) Vascular normalization in Rgs5-deficient tumours promotes immune destruction. Nature 453: 410-414.

Hanahan D, Weinberg RA (2011) Hallmarks of cancer: the next generation. Cell 144: 646-674.

Hayasaka H, Taniguchi K, Fukai S, Miyasaka M (2010) Neogenesis and development of the high endothelial venules that mediate lymphocyte trafficking. Cancer Sci 101: 2302-2308.

Hiraoka N, Onozato K, Kosuge T, Hirohashi S (2006) Prevalence of FOXP3 ${ }^{+}$ regulatory $\mathrm{T}$ cells increases during the progression of pancreatic ductal adenocarcinoma and its premalignant lesions. Clin Cancer Res 12: 5423-5434.

Hiraoka N, Petryniak B, Nakayama J, Tsuboi S, Suzuki M, Yeh JC, Izawa D, Tanaka T, Miyasaka M, Lowe JB, Fukuda M (1999) A novel, high endothelial venule-specific sulfotransferase expresses 6-sulfo sialyl Lewis(x), an L-selectin ligand displayed by CD34. Immunity 11: 79-89.

Hruban RH, Boffetta P, Hiraoka N, Iacobuzio-Donahue C, Kato Y, Kern SE, Klimstra DS, Kloppel G, Maitra A, Offerhaus GJA, Pitman MB (2010) Ductal adenocarcinoma of the pancreas. In World Health Organization Classification of Tumours. Pathology \& Genetics. Tumours of the Digestive System, Bosman FT, Carneiro F, Hruban RH, Theise ND (eds) 4th edn, pp 281-291. IARC Press: Lyon, France.

Ino Y, Yamazaki-Itoh R, Shimada K, Iwasaki M, Kosuge T, Kanai Y, Hiraoka N (2013) Immune cell infiltration as an indicator of the immune microenvironment of pancreatic cancer. Br J Cancer 108: 914-923.

Martinet L, Garrido I, Filleron T, Le Guellec S, Bellard E, Fournie JJ, Rochaix P, Girard JP (2011) Human solid tumors contain high endothelial venules: association with T- and B-lymphocyte infiltration and favorable prognosis in breast cancer. Cancer Res 71: 5678-5687.

Miyasaka M, Tanaka T (2004) Lymphocyte trafficking across high endothelial venules: dogmas and enigmas. Nat Rev Immunol 4: 360-370.

Nelson BH (2010) CD20 + B cells: the other tumor-infiltrating lymphocytes. J Immunol 185: 4977-4982.
Olive KP, Jacobetz MA, Davidson CJ, Gopinathan A, McIntyre D, Honess D, Madhu B, Goldgraben MA, Caldwell ME, Allard D, Frese KK, Denicola G, Feig C, Combs C, Winter SP, Ireland-Zecchini H, Reichelt S, Howat WJ, Chang A, Dhara M, Wang L, Ruckert F, Grutzmann R, Pilarsky C, Izeradjene K, Hingorani SR, Huang P, Davies SE, Plunkett W, Egorin M, Hruban RH, Whitebread N, McGovern K, Adams J, Iacobuzio-Donahue C, Griffiths J, Tuveson DA (2009) Inhibition of Hedgehog signaling enhances delivery of chemotherapy in a mouse model of pancreatic cancer. Science 324: 1457-1461.

Peters A, Pitcher LA, Sullivan JM, Mitsdoerffer M, Acton SE, Franz B, Wucherpfennig K, Turley S, Carroll MC, Sobel RA, Bettelli E, Kuchroo VK (2011) Th17 cells induce ectopic lymphoid follicles in central nervous system tissue inflammation. Immunity 35: 986-996.

Rangel-Moreno J, Carragher DM, de la Luz Garcia-Hernandez M, Hwang JY, Kusser K, Hartson L, Kolls JK, Khader SA, Randall TD (2011) The development of inducible bronchus-associated lymphoid tissue depends on IL-17. Nat Immunol 12: 639-646.

Remark R, Alifano M, Cremer I, Lupo A, Dieu-Nosjean MC, Riquet M, Crozet L, Ouakrim H, Goc J, Cazes A, Flejou JF, Gibault L, Verkarre V, Regnard JF, Pages ON, Oudard S, Mlecnik B, Sautes-Fridman C, Fridman WH, Damotte D (2013) Characteristics and clinical impacts of the immune environments in colorectal and renal cell carcinoma lung metastases: influence of tumor origin. Clin Cancer Res 19: 4079-4091.

Sawada J, Urakami T, Li F, Urakami A, Zhu W, Fukuda M, Li DY, Ruoslahti E, Komatsu M (2012) Small GTPase R-Ras regulates integrity and functionality of tumor blood vessels. Cancer Cell 22: 235-249.

Siegel R, Ward E, Brawley O, Jemal A (2011) Cancer statistics, 2011: the impact of eliminating socioeconomic and racial disparities on premature cancer deaths. CA Cancer J Clin 61: 212-236.

Spits H, Artis D, Colonna M, Diefenbach A, Di Santo JP, Eberl G, Koyasu S, Locksley RM, McKenzie AN, Mebius RE, Powrie F, Vivier E (2013) Innate lymphoid cells - a proposal for uniform nomenclature. Nat Rev Immunol 13: 145-149.

van de Pavert SA, Mebius RE (2010) New insights into the development of lymphoid tissues. Nat Rev Immunol 10: 664-674.

von Andrian UH, Mempel TR (2003) Homing and cellular traffic in lymph nodes. Nat Rev Immunol 3: 867-878.

Yeh JC, Hiraoka N, Petryniak B, Nakayama J, Ellies LG, Rabuka D, Hindsgaul O, Marth JD, Lowe JB, Fukuda M (2001) Novel sulfated lymphocyte homing receptors and their control by a Core1 extension beta 1,3-N-acetylglucosaminyltransferase. Cell 105: 957-969.

This work is published under the standard license to publish agreement. After 12 months the work will become freely available and the license terms will switch to a Creative Commons AttributionNonCommercial-Share Alike 4.0 Unported License.

Supplementary Information accompanies this paper on British Journal of Cancer website (http://www.nature.com/bjc) 